\title{
On ACVF of a Regime Switching AR(1) Process
}

\author{
REZA HABIBI
}

\begin{abstract}
This paper considers the auto-covariance function (ACVF) of a regime switching $\mathrm{AR}(1)$ process. Two independent Markov chains governs on auto-regressive coefficient and standard deviation of white noise process. Our approach to solve this problem is to obtain the ACVF of a AR(1) model with time varying parameters and then extend this result to regime switching case. Finally, an application of our formulae in model selection is proposed.
\end{abstract}

\section{Introduction And Main Results}

Consider a first order zero mean regime switching auto-regressive RS$\operatorname{AR}(1)$ process defined by

$$
Y_{t}=\phi_{S_{t}^{1}} Y_{t-1}+\sigma_{S_{t}^{2}} Z_{t}
$$

where $\left|\phi_{r}\right| \leq|\phi|<1$ and $\sigma_{r} \leq M$ for all $r=0, \pm 1, \pm 2, \ldots$ Here, $S_{t}^{1}$ and $S_{t}^{2}$ are two independent Markov chains with the same state space $\mathfrak{S}$. Let $Z_{t}$ be a white noise process with zero mean and variance $\sigma^{2}$. In this model, we assume that $Z_{t}$ is independent of $Y_{t-1}$ and $S_{t}^{1}$ and $S_{t}^{2}$ are independent of $Y_{t-1}$ and $Z_{t}$. In this paper, we are going to derive an expression for ACVF in this non-stationary process, i.e.,

$$
\gamma_{t}(h)=\operatorname{cov}\left(Y_{t+h}, Y_{t}\right), \quad h=0, \pm 1, \pm 2, \ldots
$$

The regime switching model deals with the capturing structural changes in the underlying financial time series using the time ordered observations. An example is Cryer and Chan (2008) who applied this type of time series for modeling the stock returns. In this model, the parameters such as mean and/or volatility vary through the sample, in fact they are functions of some Markov chain processes. This models has been applied in regression analysis, Box-Jenkins time series and as well as in GARCH modeling of financial problems. An excellent reference in this field is Zivot and Wang (2006).

2010 Mathematics Subject Classification. Primary 62G20; 62F20.

Key words and phrases. Auto-covariance function; Auto-regressive model; Markov chain; Non-stationary process; Regime switching. 
To derive $\gamma_{t}(h)$, first consider a zero mean $\operatorname{AR}(1)$ process $Y_{t}^{*}$ with time varying parameters $\phi_{t}^{*}$ and $\sigma_{t}^{*}$ defined by

$$
Y_{t}^{*}=\phi_{t}^{*} Y_{t-1}^{*}+\sigma_{t}^{*} Z_{t}^{*},
$$

where $\left|\phi_{r}^{*}\right| \leq\left|\phi^{*}\right|<1$ and $\sigma_{r}^{*} \leq M^{*}$ and $Z_{t}^{*} \sim \mathrm{WN}\left(0, \sigma^{* 2}\right)$. Suppose that $E\left(Y_{t}^{*}\right)=0$ and note that

$$
v_{t}^{*}=\operatorname{var}\left(Y_{t}^{*}\right) \leq \phi^{* 2} v_{t-1}^{*}+M^{*} \sigma^{* 2} .
$$

Therefore, we see that

$$
\begin{aligned}
v_{t}^{*} & \leq M^{*} \sigma^{* 2}\left(1+\phi^{* 2}+\cdots+\phi^{* 2(t-1)}\right)+\phi^{* 2 t} v_{1}^{*} \\
& =M^{*} \sigma^{* 2} \frac{\left(1-\phi^{* 2 t}\right)}{\left(1-\phi^{* 2}\right)}+\phi^{* 2 t} v_{1}^{*} \\
& \leq M^{*} \sigma^{* 2} \frac{\left(1-\phi^{* 2 t}\right)}{\left(1-\phi^{* 2}\right)}+v_{1}^{*}=U B .
\end{aligned}
$$

Using a recursive solution, we find that

$$
\begin{aligned}
E\left(Y_{t}^{*}-\sum_{i=0}^{k} d_{i}^{* t} Z_{t-i}^{*}\right)^{2} & =\prod_{i=0}^{k} \phi_{t-i}^{* 2} E\left(Y_{t}^{* 2}\right) \\
& \leq\left|\phi^{*}\right|^{2(k+1)} U B \\
& \rightarrow 0, \quad \text { as } k \rightarrow \infty
\end{aligned}
$$

where the coefficients $d_{i}^{* t}$ are given as follows

$$
d_{i}^{* t}=\sigma_{t-i}^{*} \prod_{j=1}^{i} \phi_{t-j+1}^{*} .
$$

Therefore, with probability one, we conclude (by letting $k \rightarrow \infty$ ) that

$$
Y_{t}^{*}=\sum_{i=0}^{\infty} d_{i}^{* t} Z_{t-i}^{*}
$$

There is another way to obtain this equation. Let $B$ denote the backward operator, i.e. $B Y_{t}^{*}=Y_{t-1}^{*}$. Note that

$$
Y_{t}^{*}=\frac{1}{1-\phi_{t}^{*} B}\left(\sigma_{t}^{*} Z_{t}^{*}\right)
$$

which equals to (using Taylor expansion)

$$
Y_{t}^{*}=\sum_{i=0}^{\infty} \prod_{j=1}^{i} \phi_{t-j+1}^{*} B^{i}\left(\sigma_{t}^{*} Z_{t}^{*}\right)=\sum_{i=0}^{\infty} d_{i}^{* t} Z_{t-i}^{*} .
$$

Using the above equation, it is not difficult to see that

$$
\operatorname{cov}\left(Y_{t}^{*}, Y_{t+h}^{*}\right)=\sigma^{* 2} \sum_{i=0}^{\infty} d_{i}^{* t} d_{i+h}^{* t} .
$$


Now, we are on position to calculate the $\gamma_{t}(h)$. Define the $\sigma$-field $\digamma$ constructed using the whole information of two Markov chains up to time $t$ as follows

$$
\digamma=\sigma\left(\left\{S_{t-i}^{1}\right\}_{i=0}^{\infty},\left\{S_{t-i}^{2}\right\}_{i=0}^{\infty}\right)
$$

and let $D_{i}^{t}=\sigma\left(S_{t-i}^{2}\right) \prod_{j=1}^{i} \phi\left(S_{t-j+1}^{1}\right)$. It is easy to see that

$$
E\left(Y_{t} Y_{t+h} \mid \digamma\right)=\sigma^{2} \sum_{i=0}^{\infty} D_{i}^{t} D_{i+h}^{t} .
$$

Therefore, using total probability law, we understand that

$$
\gamma_{t}(h)=\sigma^{2} \sum_{i=0}^{\infty} E\left(D_{i}^{t} D_{i+h}^{t}\right) .
$$

However, it seems that calculating the expectation $E\left(D_{i}^{t} D_{i+h}^{t}\right)$ be hard in practice. As follows, we give a technique which simplifies calculating this mean. To this end, suppose that $U_{t}, t \geq 0$ is a Markov chain with a finite state space. To calculate $E\left(\prod_{j=1}^{p} U_{j}\right)$, we first suppose that $p=2$, then $E\left(U_{1} U_{2}\right)=E\left(U_{1} E\left(U_{2} \mid U 1\right)\right)$. Next, let $p=3$, then

$$
\begin{aligned}
E\left(U_{1} U_{2} U_{3}\right) & =E\left(U_{1} U_{2} E\left(U_{3} \mid U_{1}, U_{2}\right)\right) \\
& =E\left(U_{1} U_{2} E\left(U_{3} \mid U_{2}\right)\right): \text { Markov property } \\
& =E\left(U_{1} E\left(U_{2} \mid U_{1}\right) E\left(U_{3} \mid U_{2}\right)\right) .
\end{aligned}
$$

Since the conditional distributions exist then this expectations can be calculated. For other choices of $p$ the same method is applied. This technique is applicable for $E\left(\prod_{j=1}^{p} h_{j}\left(U_{j}\right)\right)$ for some measurable functions $h_{j}$, $j=1,2, \ldots, p$, for more description on this method, see Iacus (2008). Note that, in practice, we can estimate $E\left(D_{i}^{t} D_{i+h}^{t}\right)$ using a Monte Carlo technique with adding some variance reduction methods, see Brazzale et al. (2007).

\section{Application}

In this section, we use an application of formulae obtained in the previous section. As we see, the ACVF is a function of expectation of some product of Markov chains. As, it is seen this expectation decays exponentially, as $h \rightarrow \infty$. Therefore, this property is transferred to ACVF. Up to this time, $\gamma_{t}(h)$ behaves like the ACVF of a ordinary $\mathrm{AR}(1)$. However, the rate of decay to zero is different form time point $t$ to $t+1$. Therefore, an unified strategy for selecting a RS-AR(1) is to monitor the time series plot of a series and if it seems there are some changes in its level, mean or variance, and if there is an exponential-wise decay to zero with different rates, then select an RS-AR model and therefore, estimate the related model parameters and do suitable statistical inference. 


\section{REFERENCES}

[1] A.R. Brazzale, A.C. Davison and N. Reid, Applied Asymptotic Case Studies in SmallSample Statistics, Cambridge University Press, 2007.

[2] J.D. Cryer and K.S. Chan, Time Series Analysis: With Applications in R, 2nd Edition, Springer Science + Business Media, New York, 2008.

[3] S.M. Iacus, Simulation and Inference for Stochastic Differential Equations: With $R$ Examples, Springer Science + Business Media, New York, 2008.

[4] E. Zivot and J. Wang, Modeling Financial Time Series with S-PLUS, Second edition, Springer Science + Business Media, New York, 2006.

\section{Reza Habibi}

Department of Statistics

Central Bank of Iran

IRAN

E-mail address: habibi1356@gmail.com 